hyperplasia) is replaced by adipose tissue in the same manner as that in the normal thymus. ${ }^{3,4}$ Pathogenesis of the tumor in our case was consistent with this theory. Third, the mixed-tumor theory proposes a mixed neoplasm of mesenchymal and endodermal origins. ${ }^{5}$ This type is thought to represent typical thymolipoma with an island of noninvoluted thymic tissue in adipose tissue. Finally, the involuting thymoma theory posits fatty degeneration of thymoma occurring in the same way as fatty replacement. ${ }^{6}$

Interestingly, the thymolipoma in our case seemed to produce CA19-9, a protein originally identified on the surface of colon cancer cells by using murine monoclonal antibodies. ${ }^{7}$ CA19-9 is a common tumor marker for gastrointestinal or pancreatic malignancy. Small amounts of CA19-9 are also present in normal cells, such as in the pancreatic, biliary, and salivary gland ducts and the prostate and bronchial glands. ${ }^{8}$ In our case the suspicion that CA19-9 was produced by the thymolipoma was confirmed on the basis of (1) preoperative absence of either gastrointestinal or pancreatic abnormalities, (2) postoperative normalization of serum CA 19-9 levels, and (3) immunoperoxidase staining with anti-CA 19-9 monoclonal antibody demonstrating CA19-9-positive epithelial cells, Hassall corpuscles, and normal thymic tissue.

Although the mechanisms behind CA19-9 production within the thymus remain unclear, production of CA19-9 within normal thymic tissue was suggested. Few reports have suggested a relationship between the thymus and CA19-9. ${ }^{9}$ In our case neoplastic growth of the thymus caused activation of CA19-9 production, thus increasing serum CA19-9 levels. Studies of larger case series are necessary to elucidate the relationships between thymolipoma and CA19-9.

\section{References}

1. Teplick JG, Nedwick A, Haskin ME. Roentgenographic features of thymolipoma. Am J Roentgenol Radium Ther Nucl Med. 1973;117: 873-7.

2. Dunn BH, Frkovich G. Lipomas of the thymus gland. Am J Pathol. 1956;32:41-51.

3. Rubin M, Mishkin S. The relationship between mediastinal lipomas and the thymus. J Thorac Surg. 1954;27:494-502.

4. Pan $\mathrm{CH}$, Chiang CY, Chen SS. Thymolipoma in patients with myasthenia gravis: report of two cases and review. Acta Neurol Scand. 1988;78:16-21.

5. Hall GFM. A case of thymolipoma with observations on a possible relationship to intrathoracic lipomata. Br J Surg. 1948;36:321-4.

6. Yamanoi S. Zur Lehre der Thymuslipome. Zentralbl Chir. 1921;48: 785-7.

7. Koprowski H, Steplewski Z, Mitchell K, Herlyn M, Herlyn D, Fuhrer P. Colorectal carcinoma antigens detected by hybridoma antibodies. Somat Cell Genet. 1979;5:957-71.

8. Okabayashi K, Motohiro A, Ueda H, Ondo K, Kawahara K, Shirakusa T. Subcarinal bronchogenic cyst with high carbohydrate antigen 19-9 production. Jpn J Thorac Cardiovasc Surg. 2002;50:46-8.

9. Aubry J, Cohen-Kaminsky S, Berrih-Aknin S. Differential expression of carcinoembryonic antigens and non cross-reacting antigens in the human thymus. Analysis on frozen sections and cultured epithelial cells using monoclonal antibodies. Thymиs. 1990;15:107-23.

\title{
The robotic, 2-stage, 3-field esophagolymphadenectomy
}

\author{
Kemp H. Kernstine, MD, PhD, ${ }^{a}$ Daniel T. DeArmond, MD, ${ }^{a}$ Mohsen Karimi, MD, ${ }^{a}$ Timothy L. Van Natta, MD, ${ }^{a}$ \\ Javier C. Campos, MD, ${ }^{b}$ Mary R. Yoder, RN, ${ }^{c}$ and Jeffrey E. Everett, MD, ${ }^{a}$ lowa City, lowa
}

$\mathrm{M}$ inimally invasive esophagectomies have been performed with low morbidity and mortality. ${ }^{1}$ Advancements in robotic engineering have allowed a robotic transhiatal esophagectomy to be performed. ${ }^{2}$ We report our initial experience with the da Vinci Surgical System (Intuitive Surgical, Inc, Sunnyvale, Calif) to perform a thoracic esophagectomy with a cervical, wide thoracic, and

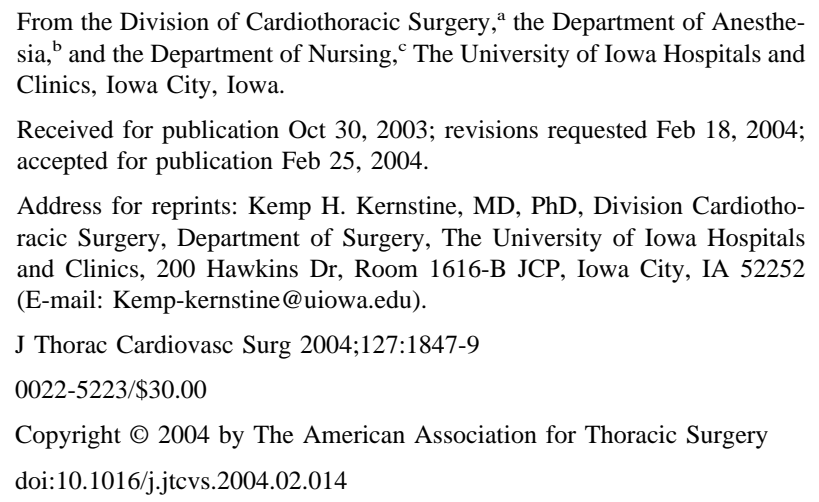

celiac axis lymphadenectomy; thoracic duct resection and ligation; creation of a gastric tube; and jejunostomy feeding tube placement with an esophagogastric anastomosis in the left side of the neck.

\section{Clinical Summary}

The patient is a 59-year-old man with a 2-month history of severe dysphagia, 40-lb weight loss, and a partially obstructing, ulcerated esophageal adenocarcinoma at 32 to $38 \mathrm{~cm}$ from the incisors with Barrett metaplasia. He had a smoking history of 80 pack-years and moderately reduced pulmonary function. The patient was preoperatively judged to have stage $\mathrm{T} 3 \mathrm{~N} 0$ disease by computed tomography, fluorodeoxyglucose positron emission tomography, and endoscopic ultrasonography. The esophagus was dilated, and the patient was treated with a 5-week induction chemoradiation program of paclitaxel, carboplatin, and 40 Gy of radiation therapy. Five weeks after the last radiation dose, a robotic esophagectomy was performed.

\section{Operative Technique}

The operation was performed in 2 stages during the same period of anesthesia, first in the left-lateral-to-nearly-prone position and then 


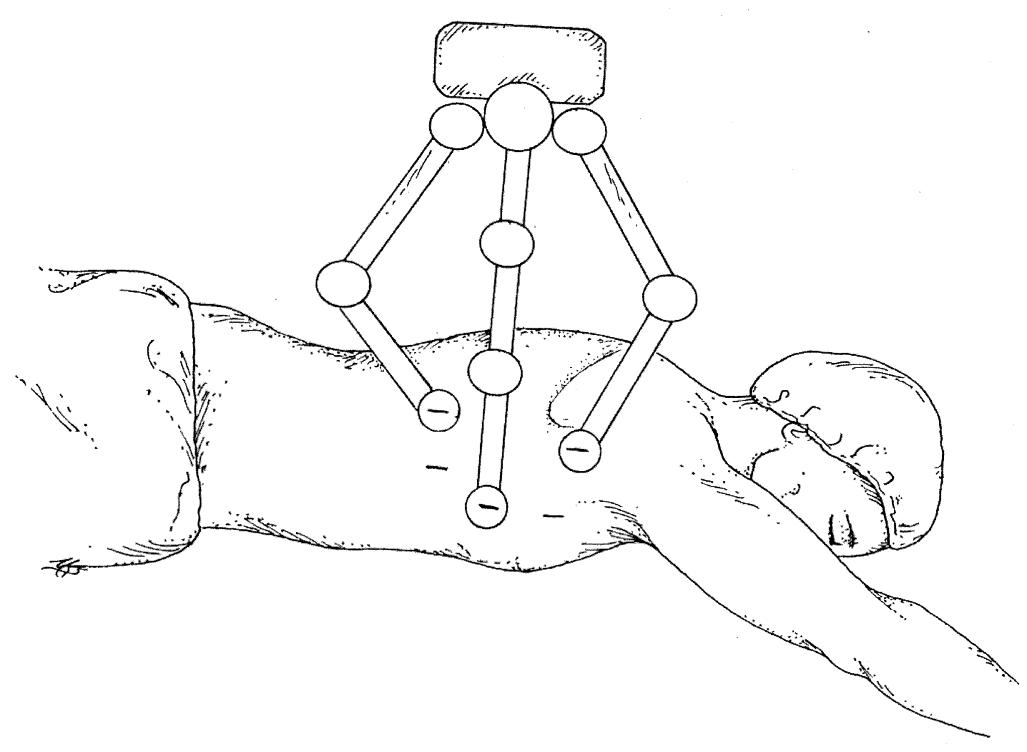

Figure 1. Stage 1.

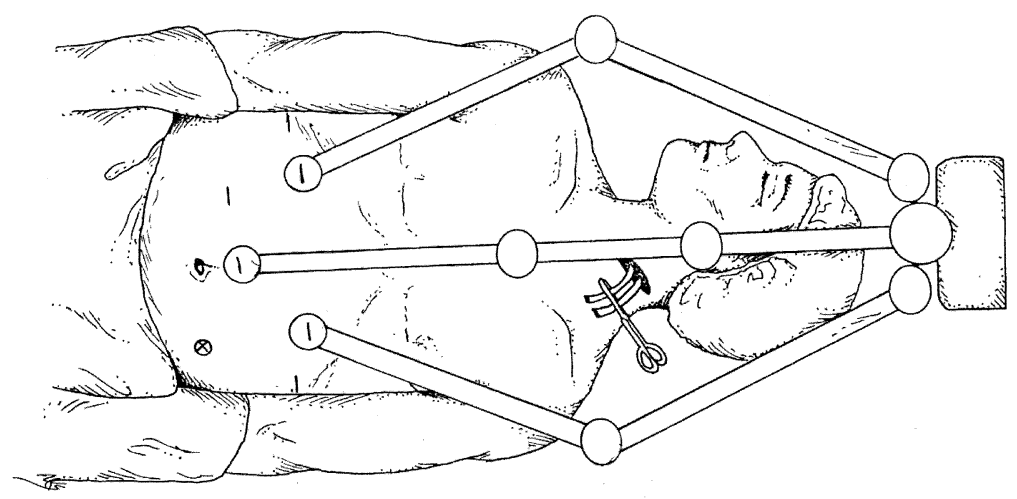

Figure 2. Stage 2.

supinely. The patient was intubated with a double-lumen endotracheal tube. His well-padded right arm was positioned over the ear with the elbow below the horizontal level of the right shoulder, and the table was tilted as far anteriorly as possible, so that the patient was $30^{\circ}$ from prone. Five puncture wounds were made in the right midanterolateral chest (Figure 1). A 12-mm trocar was placed at the fifth to sixth intercostal space in the anterior axillary line. A second port $(8 \mathrm{~mm})$ was placed in the posterior axillary line just anterior to the scapula at the third intercostal space. A third port ( 8 $\mathrm{mm}$ ) was placed in the seventh to eighth intercostal space in the posterior axillary line. The robot was brought into position, and the robotic arms and the $0^{\circ}$ videoscope were placed. Two accessory ports were placed, both at the level of the anterior axillary line, a $5-\mathrm{mm}$ port at the level of the third intercostal space and a 12-mm port at the level of the sixth to seventh intercostal space. The upper port was used for suctioning and grasping instruments, and the lower port was used to place a fan retractor for the lung. Carbon dioxide was insufflated to $10 \mathrm{~mm} \mathrm{Hg}$ of pressure to evacuate cautery smoke and to compress the lung away from the operative area.

The dissection was initiated along the anterior aspect of the esophagus at the pericardial bulge just cephalad to the inferior pulmonary vein. The resection included the esophagus and the periesophageal nodes, the adjacent thoracic duct, both hilar nodal groups, and the peritracheal nodes, leaving the cleaned azygos vein intact and continuing the dissection into the thoracic inlet to the level of the inferior laryngeal cartilage. At the level of the diaphragm, the thoracic duct was triply ligated, encompassing all of the tissue between the azygos vein and the aorta with 2-0 Ethibond suture (Ethicon, Inc, Cincinnati, Ohio) on an SH needle.

The patient was then positioned supinely and reintubated with a single-lumen endotracheal tube. The head was turned upward and to the right, exposing the left side of the neck. The upper extremities were placed by the side, and the legs were kept together. Through a 5-cm transverse incision in the left side of the neck, the esophagus was encircled with a Penrose drain, and the 
incision was packed with an antibiotic-soaked surgical sponge to prevent carbon dioxide from later escaping from the peritoneal cavity.

Next the abdominal phase was performed. Through 6 abdominal puncture wounds (Figure 2), 2 robotic arms, and the robotic viewing port, two 5-mm ports were placed beneath each costal margin in the anterior axillary line, and a $12-\mathrm{mm}$ port was placed at the left midclavicular line at the umbilicus level. The 2 robotic arms through 8 -mm ports were placed $8 \mathrm{~cm}$ subcostally at the right and left midclavicular lines. The viewing port was placed just cephalad to the umbilicus, maintaining a distance of 10 to $12 \mathrm{~cm}$ from either of the robotic arms. The abdomen was inflated to a carbon dioxide pressure of $15 \mathrm{~mm} \mathrm{Hg}$. A liver retractor exposing the esophageal hiatus was placed through the right 5-mm accessory port. The robotic arms and viewing mechanism were then placed. By using 10 firings of an Ethicon $45 \mathrm{~mm} \times 4.1 \mathrm{~mm}$ linear endostapler (Ethicon Endo-Surgery, Inc, Cincinnati, Ohio, a Johnson and Johnson Company, New Brunswick, NJ), a 4-cm wide (from the greater curvature) right gastroepiploic arterial arcadesupplied gastric tube was created without a Kocher maneuver or pyloroplasty. A laparoscopic small-bowel feeding tube (Ross Flexiflow Lap J 10F; Ross Products Division, Abbott Laboratories, Columbus, Ohio) was placed $20 \mathrm{~cm}$ from the ligament of Treitz with transcutaneously placed $\mathrm{T}$ fasteners. The most cephalad aspect of the gastric tube was then sutured to the most distal aspect of the specimen with 2 widely spaced figure-of- 8 sutures. The ventilator and chest drain suction was briefly discontinued, and the Penrose drain encircling the esophagus at the neck was used as a handle to pull the specimen up through the neck incision. Then the intraperitoneal portion of the anterior gastric conduit serosa was sutured to each esophageal hiatus crus with 2 figure-of- 8 sutures to reduce the risk of later periconduit herniation.

At the incision in the left side of the neck, the specimen was transected, and a stapled esophagogastrostomy was performed, a side-to-side functional end-to-end anastomosis with a $45 \mathrm{~mm} \times$ $4.1 \mathrm{~mm}$ linear endostapler and a $60 \mathrm{~mm} \times 4.1 \mathrm{~mm}$ transverse stapler, creating a 4 to $5 \times 6 \mathrm{~cm}$ triangular anastomosis, placing the nasogastric tube through the anastomosis before it was completed. A drain was placed in the neck beneath the platysma, and the wound was closed with interrupted absorbable sutures. Time in the operating room was 11 hours, and the total surgical console time was 4 hours and 20 minutes. Estimated blood loss was 900 $\mathrm{mL}$, and the patient received 2 units of packed cells.

The nasogastric tube was left in place for 6 days, at which time an esophagogram was performed. No anastomotic leak was found. The patient's diet was advanced to 5 small feedings per day. When we were certain there was no leak, the neck and chest drains were subsequently removed. He was discharged home on the eighth postoperative day. Only acetaminophen (INN: paracetamol) was taken for pain on postoperative day 2, and the patient was discharged home receiving acetaminophen. He is now doing well 6 months after the operation.

\section{Discussion}

Minimally invasive esophageal surgery, compared with conventional open procedures, potentially offers patients less morbidity. There is controversy over whether there is sufficiently wide periesophageal tissue resection through minimally invasive techniques to provide an adequate cancer operation. Esophagectomy has been a technically demanding procedure with a relatively high morbidity and mortality. ${ }^{3}$ As a sole means of cancer treatment, it appears to have limited utility, with fewer than $10 \%$ of patients surviving for 5 years. Minimally invasive techniques appear to improve the surgical morbidity, but their ability to achieve similar or superior cure rates to those of the open technique remains to be proved. With the advent of robotics, the increased magnification, dexterity, and 3-dimensional visual clarity allow one to precisely and reproducibly perform esophageal resection en bloc, along with periesophageal tissue potentially harboring malignancy.

There is a prior case report of a robotic esophagectomy. ${ }^{2}$ The esophagus was removed in a transhiatal fashion. No wide nodal resection was performed because the robotic arms are too short to reach the carina, requiring the peritracheal portion of the esophagus to be removed through the neck incision and thoracic inlet. As stated by the authors, their procedure is limited to a restricted few patients, those with very limited disease or severe dysplasia. Our technique applies to a broader group of patients. In either case, the ability to accurately stage and improve survival remains to be discerned and should be evaluated in a prospective trial.

Development of an operating room team is absolutely critical to minimize operating room time. The surgical assistant provides retraction, assistance in mobilization, and suctioning at precise times. An experienced anesthesiologist who can perform singlelung ventilation and hemodynamically support the patient and an operating nurse and support nursing staff are critical to maintain the flow of the procedure. In this case report, more than half of the operating time was nonsurgical.

This is the first reported case of a 2-stage, 3-field robotic esophagolymphadenectomy for advanced-stage disease. With improvements in robotic equipment, superior visualization, and development of a robotics team, we expect that this minimally invasive wide resection of the esophagus en bloc with the periesophageal tissue could potentially provide an oncologically superior resection, yet reduce the encumbrance to the patient.

\section{References}

1. Nguyen N, Schauer P, Luketich J. Minimally invasive esophagectomy for Barrett's esophagus with high grade dysplasia. Surgery. 2000;127: 284-90.

2. Horgan S, Berger RA, Elli EF, Espat NJ. Robotic-assisted minimally invasive transhiatal esophagectomy. Am Surg. 2003;69:624-6.

3. Hulscher JB, Tijssen JG, Obertop H, van Lanschot JJ. Transthoracic versus transhiatal resection for carcinoma of the esophagus: a metaanalysis. Ann Thorac Surg. 2001;72:306-13. 